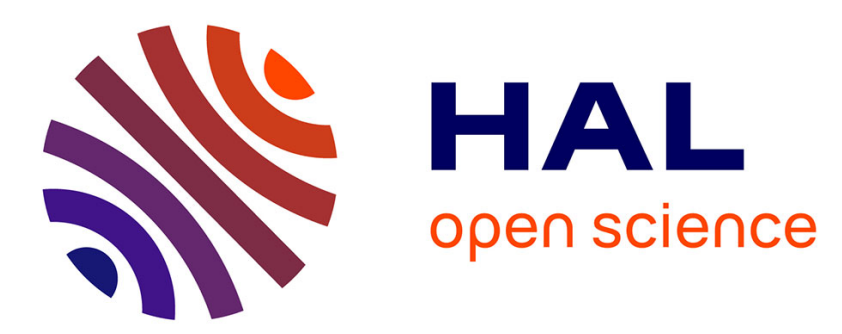

\title{
3D fluid-solid coupled full-waveform inversion for ocean-bottom seismic data
}

\author{
Jian Cao, Romain Brossier, Ludovic Métivier
}

\section{To cite this version:}

Jian Cao, Romain Brossier, Ludovic Métivier. 3D fluid-solid coupled full-waveform inversion for oceanbottom seismic data. First International Meeting for Applied Geoscience \& Energy, Sep 2021, Denver, United States. pp.832-836, 10.1190/segam2021-3583300.1 . hal-03404536

\section{HAL Id: hal-03404536 \\ https://hal.science/hal-03404536}

Submitted on 26 Oct 2021

HAL is a multi-disciplinary open access archive for the deposit and dissemination of scientific research documents, whether they are published or not. The documents may come from teaching and research institutions in France or abroad, or from public or private research centers.
L'archive ouverte pluridisciplinaire HAL, est destinée au dépôt et à la diffusion de documents scientifiques de niveau recherche, publiés ou non, émanant des établissements d'enseignement et de recherche français ou étrangers, des laboratoires publics ou privés. 


\section{D fluid-solid coupled full waveform inversion for ocean-bottom seismic data}

Jian Cao ${ }^{1 *}$, Romain Brossier ${ }^{1}$, Ludovic Métivier ${ }^{1,2}$

${ }^{1}$ Univ. Grenoble Alpes, ISTerre, F-38058 Grenoble, France

${ }^{2}$ Univ. Grenoble Alpes, CNRS, LJK, F-38058 Grenoble, France

\section{SUMMARY}

Ocean-bottom seismic acquisition is attractive for the exploration of challenging marine environments. Compared with conventional streamer acquisitions, its separation of sources and receivers makes a significant improvement in terms of illumination, especially at depth. Furthermore, such acquisition system makes it possible to record fourcomponent data through a combination of hydrophones and threecomponent (3C) geophones. The information on elastic properties of the subsurface is better captured by those $3 \mathrm{C}$ geophones on the seabed. This information is mostly overlooked up to now, while reconstructing jointly $\mathrm{P}$-wave and $\mathrm{S}$-wave velocity models would significantly improve the subsurface characterization. To achieve such a highresolution multi-parameter reconstruction, we design an efficient 3D fluid-solid coupled full waveform inversion (FWI) engine. It is based on the acoustic-elastic coupled wave-equation system, in which fluid and solid domains are divided explicitly and handled with acousticand elastic-wave equations, respectively. A hybrid approach for the misfit gradient building is proposed in such fluid-solid coupled FWI, in which multi-parameter gradient kernels, including the one related to $\mathrm{S}$-wave velocity, are constructed by using a similar modeling solver in both forward and adjoint simulations. This FWI engine is illustrated on a bilayered 2D model and a 3D extended Marmousi model. We show how P-wave and S-wave velocity models can be inferred from the data, and that the resolution improvement can be obtained from the reconstruction of the S-wave velocity model, which highlights the important contribution of $3 \mathrm{C}$ geophone dataset.

\section{INTRODUCTION}

As the marine seismic exploration moves to complex deep-water geologic environments, ocean-bottom seismic acquisition (either OBC - ocean-bottom cables or OBN - ocean-bottom nodes) begins to be used as an effective technology for imaging quality enhancement and risk reduction. Compared with streamer acquisition, ocean-bottom acquisition has the following two advantages: 1) separating sources from receivers to achieve a wide-azimuth coverage and long sourcereceiver distance for improving the illumination at depth, 2) providing richer subsurface information through hydrophones in the water and $3 \mathrm{C}$ geophones on the seabed which can better record elastic effects for a precise S-wave velocity reconstruction (Maver 2011).

Full waveform inversion (FWI) is a powerful technique to extract highresolution quantitative physical parameters of the subsurface by fitting the full information of seismic data (Lailly 1983, Tarantola 1984 Virieux and Operto 2009, Virieux et al. 2017). In marine environments, most FWI studies are developed in the acoustic approximation (with or without anisotropy and attenuation) using the hydrophone dataset only (Sirgue et al. 2010, Morgan et al. 2013, Prieux et al. 2013a Operto et al. 2015. Amestoy et al. 2016, Wang et al. 2019 Kamath et al. 2020). This simplified physics implies that the solid Earth is viewed as a fluid, ignoring its elastic properties. Although elastic effects in many hydrophone datasets are weak due to sources and receivers typically in the water (which is not the case for 3C geophones in the ocean-bottom acquisition) and limited time window recording essentially P-wave phases, they can still be observed on the $\mathrm{P}$-wave amplitudes (AVO effect), and therefore have a direct impact on the acoustic FWI (Barnes and Charara 2009). To mitigate elastic effects, several methods have been developed, such as the use of Wiener filters and estimated $V_{p} / V_{s}$ models to convert elastic data into pseudoacoustic data in the acoustic FWI (Agudo et al. 2018 2020), or more recently the use of machine learning to obtain an acoustic version of the elastic data in the data processing (Yao et al. 2020). Those methods aim at improving the stability of P-wave velocity reconstruction in the (visco-)acoustic approximation, disregarding the S-wave veloc- ity reconstruction, which significantly reduces the computational cost of FWI. However, reconstructing jointly P-wave and S-wave velocity models would significantly improve the subsurface characterization, especially for the indication of hydrocarbon reservoirs (Sears et al. 20082010 Prieux et al. 2013b. To better mimic the wave propagation physics and use the elastic information from $3 \mathrm{C}$ geophones in the ocean-bottom acquisition, the FWI based on an elastic approximation for the subsurface maybe worth the extra computational cost.

Here we aim at developing a flexible and efficient 3D fluid-solid coupled FWI engine to achieve a high-resolution multi-parameter reconstruction of the subsurface. For reducing computational cost and memory requirement, we consider only using the elastic wave equation in the subsurface and the wave propagation in the above water layer is still modeled by the acoustic wave equation. Consequently, the forward problem in the fluid-solid coupled medium is described by an acoustic-elastic coupled wave-equation system, and the corresponding methodology of FWI gradient building is developed based on the adjoint-state method (Plessix 2006). A time-domain spectral-element method (SEM) with a flexible 3D Cartesian-based hexahedral mesh is used for its numerical implementation. An accurate coupling of the acoustic- and elastic-wave equations is implemented and high computational efficiency is achieved through the domain-decomposition based parallelization. In the following sections, we first review the acoustic-elastic coupled wave-equation system for formulating the fluidsolid coupled problem, which is followed by the definition of the associated FWI problem and the gradient building with a hybrid approach. Then, we validate the proposed hybrid approach for gradient building on a fluid-solid bi-layered model, and show how P-wave and S-wave velocity models can be inferred from data. Finally, we present a fluidsolid coupled FWI case study on a 3D extended Marmousi model to reveal what we can benefit from the reconstruction of S-wave velocity model and $3 \mathrm{C}$ geophone data using an ocean-bottom acquisition.

\section{METHODOLOGY}

In the forward problem, the acoustic-elastic coupled wave-equation system is commonly formulated in terms of fluid potential $\varphi$ and solid displacement $\mathbf{u}_{s}\left(\varphi-\mathbf{u}_{s}\right.$ formulation) Chaljub and Valette 2004, Peter et al. 2011), due to its good performance of accuracy and efficiency in the modeling (Cao et al. 2020a b). This system writes

$$
\begin{array}{ll}
\frac{1}{\kappa} \ddot{\varphi}-\nabla \cdot\left(\frac{1}{\rho_{f}} \nabla \varphi\right)=\frac{1}{\kappa} \iint-P_{f} d t d t, & \text { in } \Omega_{f}, \\
\rho_{s} \ddot{\mathbf{u}}_{s}=\nabla \cdot \boldsymbol{\sigma}+\mathbf{f}_{s}, \quad \boldsymbol{\sigma}=\mathbf{C}: \boldsymbol{\varepsilon}, & \text { in } \Omega_{s}, \\
\mathbf{u}_{s} \cdot \mathbf{n}=\frac{1}{\rho_{f}} \nabla \varphi \cdot \mathbf{n}, \quad \boldsymbol{\sigma}_{s} \cdot \mathbf{n}=\ddot{\varphi} \mathbf{n}, & \text { on } \Gamma_{f s},
\end{array}
$$

where $\Omega_{f}$ and $\Omega_{s}$ denote the fluid and solid regions, respectively, $\Gamma_{f s}$, which can be any continuous curvilinear shape, denotes the fluid-solid interface with a unit normal vector $\mathbf{n}$, and $\boldsymbol{\sigma}$ and $\boldsymbol{\varepsilon}$ are the stress and strain tensors in the solid region. The fluid part is parameterized with density $\rho_{f}$ and bulk modulus $\kappa$, while the solid part is parameterized with density $\rho_{s}$ and elastic stiffness tensor $\mathbf{C}$. The source term can be applied in the fluid region in terms of pressure $P_{f}$ or in the solid region as a body force $\mathbf{f}_{s}$.

FWI applied to ocean-bottom seismic data writes

$$
J(\mathbf{m})=\frac{1}{2} \sum_{s, r}\left(\alpha\left\|\mathbf{S}_{s, r} \mathbf{W}_{P}-\mathbf{d}_{P_{s, r}}^{o b s}\right\|^{2}+\beta\left\|\mathbf{S}_{s, r} \mathbf{W}_{\mathbf{u}}-\mathbf{d}_{\mathbf{u}_{s, r}}^{o b s}\right\|^{2}\right),
$$

where $\mathbf{S}_{s, r}$ is the restriction operator extracting the full wavefields of pressure $\mathbf{W}_{P}$ and displacement $\mathbf{W}_{\mathbf{u}}$ at the receiver position $r$ for the source $s$, and $\mathbf{d}_{P_{s, r}}^{o b s}$ and $\mathbf{d}_{\mathbf{u}_{s, r}}^{o b s}$ are the corresponding observed pressure and $3 \mathrm{C}$ displacement data, respectively. The scaling factors $\alpha$ and $\beta$ are introduced for the dimensionless conversion, which contributes to avoiding the influence from the order of magnitudes of different data components. Following the conventional FWI scheme, this misfit 


\section{D fluid-solid coupled FWI}
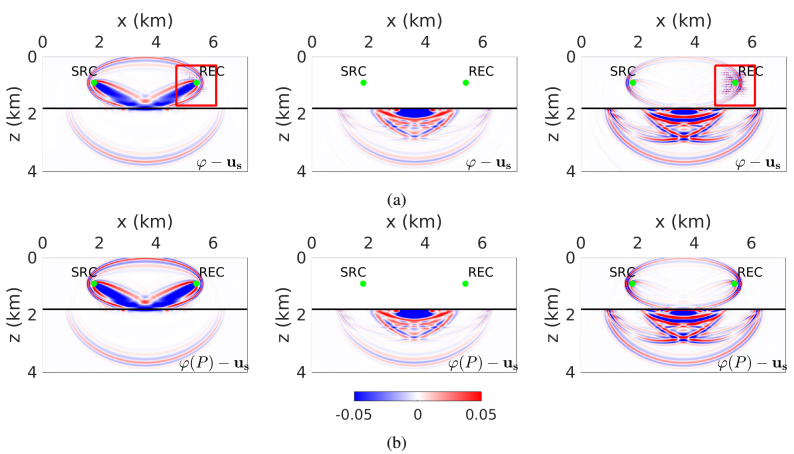

Figure 1: Comparison of gradient kernels computed by the $\varphi-\mathbf{u}_{s}$ formulation based approach (a) and the hybrid approach (b) for different parameters: P-wave velocity (left column), S-wave velocity (middle column) and density (right column). The source (SRC) and receiver (REC) denoted by green dots are both in the fluid domain.

function is minimized through local optimization methods which require to compute its gradient. The latter is obtained from the zero-lag cross-correlation of forward wavefields and adjoint wavefields, following the adjoint-state method (Plessix 2006). The adjoint wavefields in both fluid and solid domains are obtained by solving the adjoint system of the acoustic-elastic coupled wave equations:

$$
\begin{aligned}
& \frac{1}{\kappa} \ddot{\mu}^{(\varphi)}-\nabla \cdot\left(\frac{1}{\rho_{f}} \nabla \mu^{(\varphi)}\right)=-\alpha \sum_{s, r} \mathbf{S}_{s, r}^{T} \partial_{t t}\left(\mathbf{S}_{s, r} \mathbf{W}_{P}-\mathbf{d}_{P_{s, r}}^{o b s}\right), \\
& \rho_{s} \ddot{\boldsymbol{\lambda}}=\nabla \cdot \mathbf{T}+\beta \sum_{s, r} \mathbf{S}_{s, r}^{T}\left(\mathbf{S}_{s, r} \mathbf{W}_{\mathbf{u}}-\mathbf{d}_{\mathbf{u}_{s, r}}^{o b s}\right), \quad \mathbf{T}=\mathbf{C}: \nabla \boldsymbol{\lambda}, \\
& \ddot{\boldsymbol{\lambda}} \cdot \mathbf{n}=-\frac{1}{\rho_{f}} \nabla \mu^{(\varphi)} \cdot \mathbf{n}, \quad \mathbf{T} \cdot \mathbf{n}=-\mu^{(\varphi)} \mathbf{n},
\end{aligned}
$$

where the adjoint wavefields $\mu^{(\varphi)}$ and $\boldsymbol{\lambda}$ are associated with the fluid potential $\varphi$ and solid displacement $\mathbf{u}$, respectively, and symbol $\partial_{t t}$ denotes the second-order time derivative. Note that this adjoint system is different from its corresponding forward system (Eq. 1 in in the form of the boundary conditions, where an additional sign "-" and time derivatives are required. It implies that the wave modeling solver for the forward problem needs to be modified in the adjoint modeling.

To re-exploit the forward modeling solver in the solving of the adjoint system, we consider the $P-\mathbf{u}_{s}$ acoustic-elastic coupled formulation where the pressure $P$ is used in the acoustic wave equation, and then an adjoint system which has a similar structure as Eq. 11] can be obtained $\frac{1}{\kappa} \ddot{\mu}^{(P)}-\nabla \cdot\left(\frac{1}{\rho_{f}} \nabla \mu^{(P)}\right)=\alpha \sum_{s, r} \mathbf{S}_{s, r}^{T}\left(\mathbf{S}_{s, r} \mathbf{W}_{P}-\mathbf{d}_{P_{s, r}}^{o b s}\right), \quad$ in $\Omega_{f}$, $\rho_{s} \ddot{\boldsymbol{\lambda}}=\nabla \cdot \mathbf{T}+\beta \sum_{s, r} \mathbf{S}_{s, r}^{T}\left(\mathbf{S}_{s, r} \mathbf{W}_{\mathbf{u}}-\mathbf{d}_{\mathbf{u}_{s, r}}^{o b s}\right), \quad \mathbf{T}=\mathbf{C}: \nabla \boldsymbol{\lambda}, \quad$ in $\Omega_{s}$, $\boldsymbol{\lambda} \cdot \mathbf{n}=\frac{1}{\rho_{f}} \nabla \mu^{(P)} \cdot \mathbf{n}, \quad \mathbf{T} \cdot \mathbf{n}=\ddot{\mu}^{(P)} \mathbf{n}$ on $\Gamma_{f s}$.

where the adjoint wavefields $\mu^{(P)}$ is associated with the pressure $P$. Its corresponding gradient expressions of density $\rho$ and bulk modulus $\kappa$ in the fluid domain are

$$
\frac{\partial J(\mathbf{m})}{\partial \rho}=\left(\nabla \mu^{(P)}, \frac{1}{\rho^{2}} \nabla P\right)_{\Omega_{f}, t}, \frac{\partial J(\mathbf{m})}{\partial \kappa}=\left(\mu^{(P)}, \frac{1}{\kappa^{2}} \ddot{P},\right)_{\Omega_{f}, t},
$$

and the gradient expressions of density $\rho$ and elastic stiffness tensor $\mathbf{C}$ in the solid domain are

$$
\frac{\partial J(\mathbf{m})}{\partial \rho}=-(\boldsymbol{\lambda}, \ddot{\mathbf{u}})_{\Omega_{s}, t}, \quad \frac{\partial J(\mathbf{m})}{\partial C_{i j k l}}=-\left(\nabla \boldsymbol{\lambda}, \frac{\partial \mathbf{C}}{\partial C_{i j k l}}:: \nabla \mathbf{u}\right)_{\Omega_{s}, t},
$$

By combining the $\varphi-\mathbf{u}_{s}$ formulation for the forward simulation and the $P-\mathbf{u}_{s}$ formulation for the adjoint simulation, a hybrid approach is proposed to make the same modeling solver to be used in both simulations. The algorithm can be sketched as follows: (1) solve the $\varphi-\mathbf{u}_{s}$ forward system (Eq. (1) for the forward wavefields $\varphi$ in the fluid domain and $\mathbf{u}_{s}$ in the solid domain, where the required pressure wavefield is computed by $P=-\ddot{\varphi}$; (2) based on the pressure wavefield in (1), solve the $P-\mathbf{u}_{s}$ adjoint system (Eq. (4) with the same explicit modeling solver as in (1) for the adjoint wavefields $\mu^{(P)}$ in the fluid domain and $\boldsymbol{\lambda}$ in the solid domain; (3) compute the zero-lag crosscorrelation of the forward wavefields and the adjoint wavefields to get
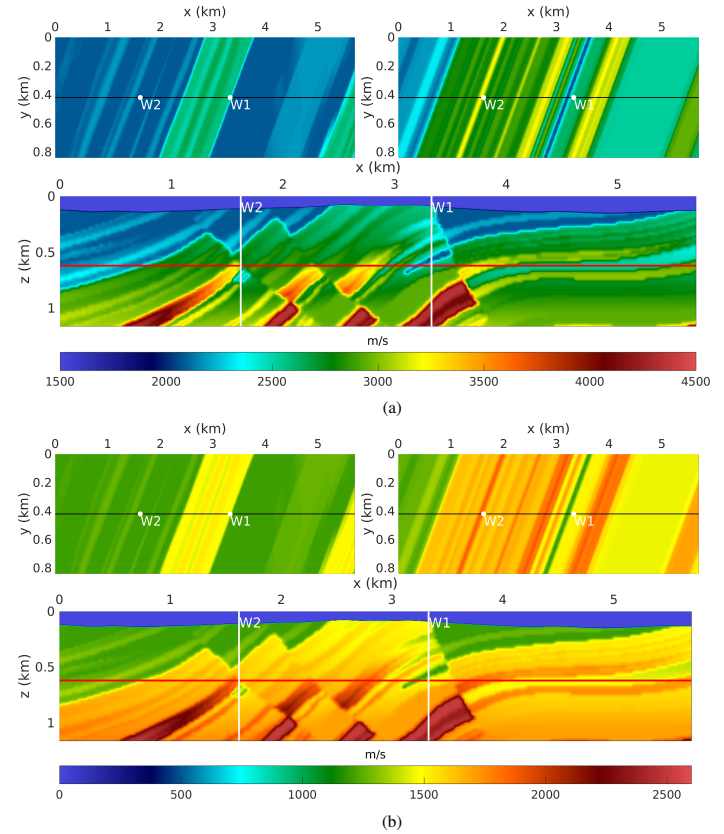

Figure 2: Velocity slices of 3D extended Marmousi-II model: P-wave velocity (a) and S-wave velocity (b). In each figure, the slices at the seabed, $z=0.62 \mathrm{~km}$ (red line) and $y=0.42 \mathrm{~km}$ (black line) are shown. $\mathrm{W} 1$ and W2 are the wells across two faulted gas traps, respectively.

the elementary gradients with the help of Eqs. 57 and (6). The gradient for any other parameters, such as $\mathrm{P}$-wave $\left(V_{p}\right)$ and $\mathrm{S}$-wave $\left(V_{s}\right)$ velocities, are obtained by chain rule.

\section{NUMERICAL EXAMPLES}

In this section, we validate the feasibility of the proposed hybrid approach in the gradient building and show the application of acousticelastic coupled FWI on a 3D extended Marmousi-II model to highlight its capability in the high-resolution multi-parameter reconstruction. All these numerical examples are based on a time-domain spectralelement discretization, thanks to its accurate implementation of boundary conditions even with irregular interfaces.

\section{Validation study}

The FWI gradient is composed of the gradient kernels from multiple source-receiver couples. Based on a simple fluid-solid bilayered 2D model, we compare the gradient kernels of a specific source-receiver couple computed in two ways: 1) only using the $\varphi-\mathbf{u}_{s}$ formulation with a redesigned numerical scheme for modeling the adjoint wavefield, (2) using the proposed hybrid approach. Here we add perturbations of $+500 \mathrm{~m} / \mathrm{s}$ on the P-wave velocity, $+200 \mathrm{~m} / \mathrm{s}$ on the $\mathrm{S}$-wave velocity and $+50 \mathrm{~kg} / \mathrm{m}^{3}$ on the density for the solid layer, and the source is a pressure source with $10 \mathrm{~Hz}$ Ricker wavelet. The resulting gradient kernels of P-wave velocity, S-wave velocity and density are illustrated in Figure 1 We can see that the model parameters can be inferred from the data through the contribution of different waves, such as the diving wave, reflected wave and P-to-S converted wave that supports the $\mathrm{S}$-wave velocity reconstruction. The gradient kernels obtained from the hybrid approach are nearly identical with the one following the $\varphi-\mathbf{u}_{s}$ formulation rigorously, confirming the feasibility of the hybrid approach in the gradient building. In addition, thanks to no additional time derivative of the data misfit in the adjoint source, the hybrid approach produces a cleaner gradient kernel than the one from the $\varphi-\mathbf{u}_{s}$ formulation which has some high-frequency noise on the receiver side (see the zones delineated by red squares in Figure 1 i).

\section{FWI application}

The application study of acoustic-elastic coupled FWI is based on a 3D extended Marmousi-II model. It is obtained by extending the 2D Marmousi-II model (Martin et al. 2006) along the $y$-direction with an angle of $45^{\circ}$. Moreover, an uneven seabed is added to replace the original flat one. We extract the slices at the seabed, the depth of $z=$ $0.62 \mathrm{~km}$ and along the crossline direction with $y=0.42 \mathrm{~km}$ for display. 


\section{D fluid-solid coupled FWI}

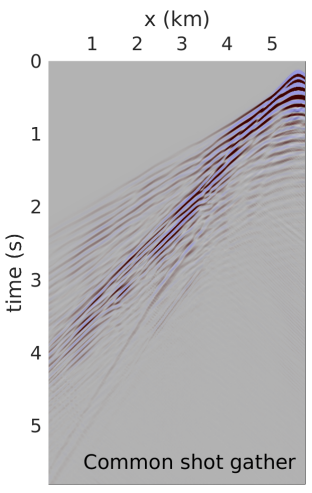

(a)

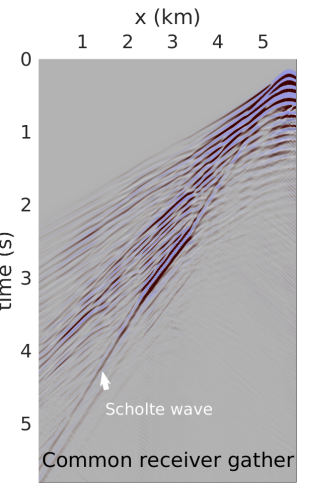

(b)

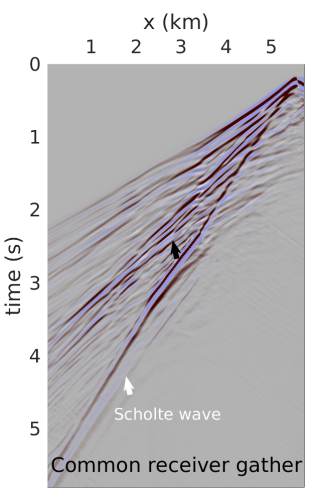

(c)

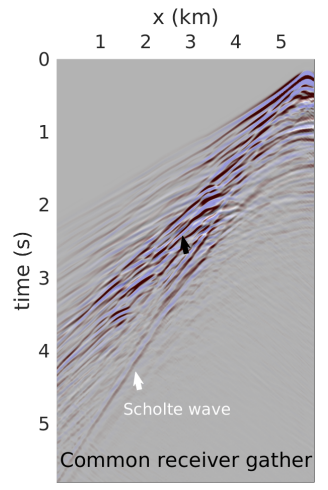

(d)

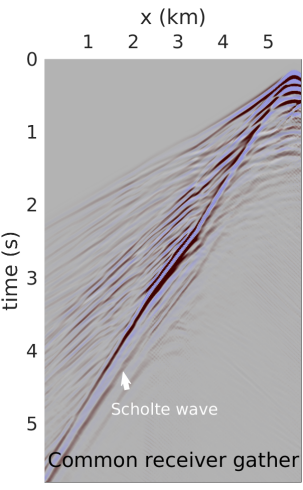

(e)

Figure 3: Data-fitting illustration at the crossline $y=0.425 \mathrm{~km}$ for pressure data in the streamer acquisition (a), pressure data in the OBC acquisition (b) and $3 \mathrm{C}$ displacement data in the $\mathrm{OBC}$ acquisition (c: $u_{x}, \mathrm{~d}: u_{y}$ and $\mathrm{e}: u_{z}$ ). The synthetic data (plotted in blue-red) are superimposed onto the observed data (plotted in white-black). The white arrows highlight the Scholte wave propagating along the seabed. The black arrows indicate the converted P-to-S energy recorded from the horizontal components, which is dominant in the $y$ component (b).

\begin{tabular}{|c|c|c|c|c|c|c|c|c|c|}
\hline \multirow{2}{*}{ Model } & \multirow{2}{*}{$\begin{array}{c}\text { Mesh } \\
(x \times y \times z)\end{array}$} & \multirow{2}{*}{$\begin{array}{l}\text { Element } \\
\text { size }(m)\end{array}$} & \multirow{2}{*}{ NT } & \multirow{2}{*}{ Iterations } & \multirow{2}{*}{ Data type } & \multirow{2}{*}{ Cores } & \multicolumn{3}{|c|}{ Run time (hour) } \\
\hline & & & & & & & Band 1 & Band 2 & Band 3 \\
\hline \multirow{3}{*}{ 3D Marmousi-II } & \multirow{3}{*}{$102 \times 21 \times 24$} & \multirow{3}{*}{60} & \multirow{3}{*}{12,000} & \multirow{3}{*}{$60 \times 3$} & Streamer & 1,520 & 3.85 & 3.90 & 4.21 \\
\hline & & & & & OBC-P & 1,520 & 3.81 & 4.12 & 4.17 \\
\hline & & & & & OBC-3C & 4,560 & 3.64 & 4.02 & 4.25 \\
\hline
\end{tabular}

Table 1: Summary of parameter settings and computational costs in the acoustic-elastic coupled FWI test. The frequency bands are : $0-5 \mathrm{~Hz}$ (Band 1), $0-10 \mathrm{~Hz}$ (Band 2), $0-20 \mathrm{~Hz}$ (Band 3). Those tests are run on Jean Zay machine from the French National supercomputing center IDRIS (HPE SGI 8600 supercomputer) with Intel Cascade Lake CPU architecture.

Two vertical profiles are also extracted across the faulted gas traps for QC measurement (see two white lines marked W1 and W2 in Figure 2). The initial $V_{p}$ and $V_{s}$ are smoothed from the true models (see Figure 4 a). A similar smoothed density model is used in both observed data generation and inversion.

We run the acoustic-elastic coupled FWI on three different datasets: (1) pressure data recorded by a towed streamer acquisition with 2 shot lines ( 38 shots, every $300 \mathrm{~m}$ in the inline and crossline directions) in which each shot is recorded by 9 streamers with streamer separation of $50 \mathrm{~m}$ and maximum offset of $5.8 \mathrm{~km}$ in the acquisition direction, (2) pressure data recorded by a OBC acquisition with 18 shot lines covering the whole $x-y$ plane and 2 ocean-bottom cables (38 4C receivers, every $300 \mathrm{~m}$ in the inline and crossline directions), (3) 3C displacement data recorded by the same OBC acquisition as in (2). The source function for generating all the datasets is a $10 \mathrm{~Hz}$ Ricker wavelet. The maximum frequency considered for inversion is $20 \mathrm{~Hz}$. The $V_{p}$ and $V_{s}$ models are inverted simultaneously with the same inversion process for each dataset, except that a source-receiver reciprocity is applied in OBC datasets for decreasing the computational cost. The detailed parameter settings and computational costs are listed in Table 1 It can be seen that CPU cores used for three-component data (OBC-3C) are three times as much as those for one-component data (OBC-P). The reason is that $3 \mathrm{C}$ geophones are viewed as $3 \mathrm{C}$ sources in the sourcereceiver reciprocity, leading to the number of source tripled in the FWI. The data-fitting illustration in Figure 3 shows that we obtain a good agreement between synthetic data and observed data in all three datasets (no blue and red). This implies successful inversion in all three cases. Thus, the reconstructed results should only be influenced by the dataset itself. Figures $4 \mathrm{p}-4 \mathrm{~d}$ show the reconstructed $V_{p}$ and $V_{s}$ models from the three datasets. As expected, due to a wide-azimuth coverage and long offset, OBC acquisition efficiently mitigates the footprint and unwanted artifacts observed in the streamer acquisition and recovers more structure details in both $V_{p}$ and $V_{s}$ (compare Figure $4 \mathrm{p}$ and 4 ). However, a better velocity reconstruction can be seen in the results from $3 \mathrm{C}$ displacement dataset (Figures $4 \mathrm{~d}$ ) where the $\mathrm{S}$ wave velocity is almost totally recovered, owing to a direct recording of S-wave on the seabed (see the converted P-to-S energy indicated by black arrows in Figures 3 , and $3 \mathrm{~d}$ ). In Figure 5 we can see that the gas trap indicated by the black arrow at location W1 is clearly recovered from all three datasets. A higher resolution is obtained from the recon- structed S-wave velocity model due to a smaller wavelength of S-wave compared with P-wave. For the W2 profile, it passes through two adjacent gas traps (low velocity anomalies indicated by black arrows). The comparison of reconstructed $V_{p}$ and $V_{s}$ models at this profile reveals that a proper distinction between two gas traps can be achieved through inverting the $V_{s}$ model from OBC datasets (either pressure or $3 \mathrm{C}$ displacement data), as indicated by the blue arrow in Figure $5 \mathrm{p}$.

\section{CONCLUSIONS}

We propose an efficient 3D acoustic-elastic coupled FWI engine which can reconstruct jointly $\mathrm{P}$-wave and $\mathrm{S}$-wave velocity models from multicomponent ocean-bottom data. It is developed within the framework of acoustic-elastic coupled wave-equation system, based on an elastic approximation of the subsurface. From the adjoint-state method, the gradient kernels of this acoustic-elastic coupled FWI are constructed using a hybrid approach. It involves two different formulations for representing the acoustic-elastic coupled wave-equation system (the $\varphi-$ $\mathbf{u}_{s}$ formulation for modeling the forward wavefield, and the $P-\mathbf{u}_{s}$ formulation for modeling the adjoint wavefield), which makes the same wave propagation solver can be used in the solution of both forward and adjoint problems. The comparison of gradient kernels on the bilayered 2D model illustrates the feasibility of the hybrid approach in the gradient building and its advantage regarding the elimination of unwanted high-frequency noise on the receiver side. In the 3D extended Marmousi-II model case study, the application of acoustic-elastic coupled FWI to the multi-component OBC data reveals that a significant resolution improvement can be obtained from the reconstruction of the $\mathrm{S}$-wave velocity model, especially from $3 \mathrm{C}$ geophone data.

\section{ACKNOWLEDGEMENTS}

The research leading to these results has received funding from the European Union's Horizon 2020 research and innovation programme under the ENERXICO project, grant agreement No. 828947. This study was also partially funded by the SEISCOPE consortium (http:// seiscope2.osug.fr, sponsored by AKERBP, CGG, CHEVRON, EQUINOR, EXXON-MOBIL, JGI, SHELL, SINOPEC, SISPROBE and TOTAL. This study was granted access to the HPC resources of CIMENT infrastructure (https://ciment.ujf-grenoble.fr), Cray Marketing Partner Network (https://partners.cray.com) and CINES/IDRIS/TGCC under the allocation 046091 made by GENCI 


\section{D fluid-solid coupled FWI}
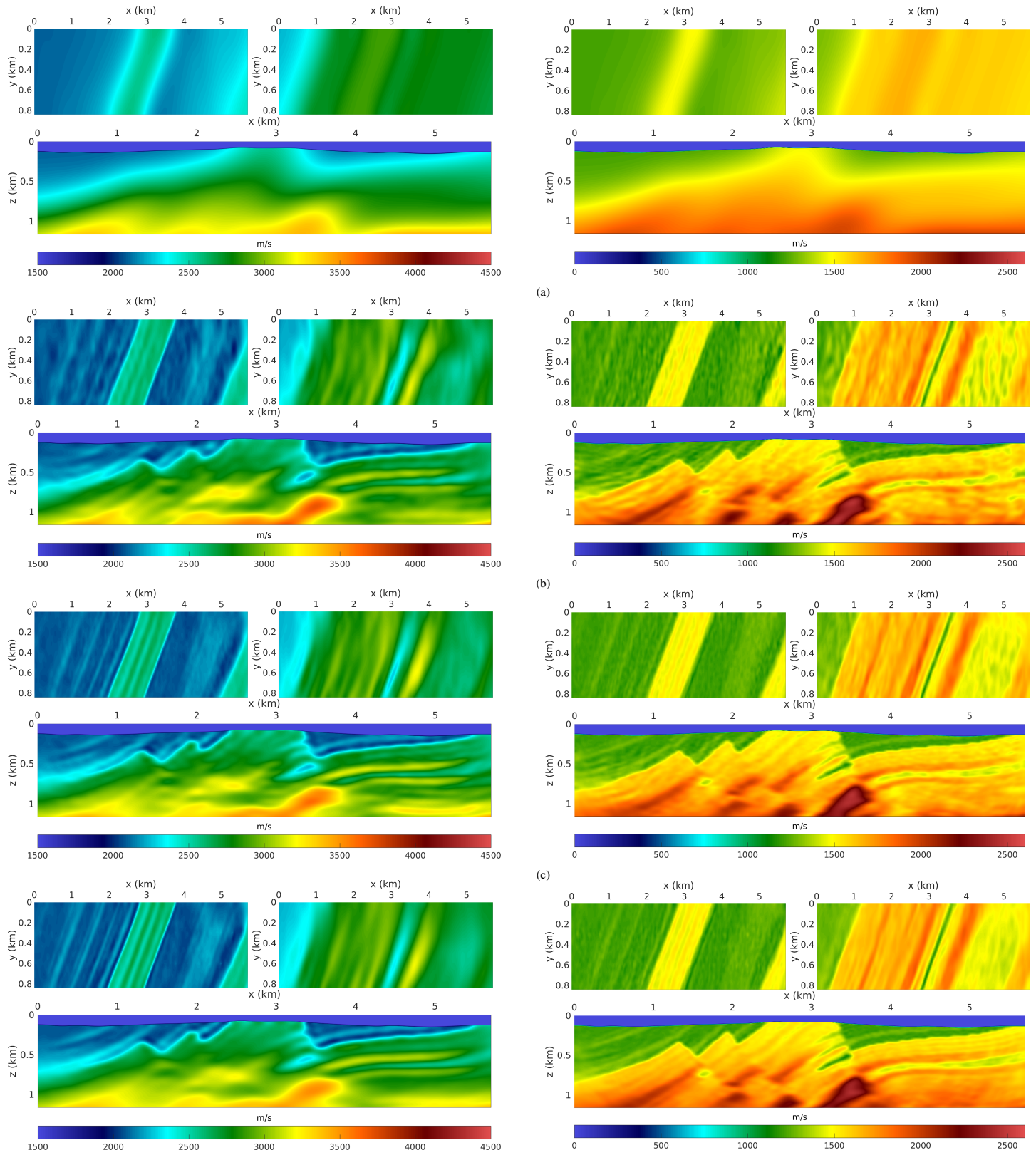

Figure 4: Initial models (a) and reconstructed models of P-wave velocity (left column) and S-wave velocity (right column) by using the streamer acquisition (b), OBC acquisition with pressure data only (c) and $\mathrm{OBC}$ acquisition with $3 \mathrm{C}$ displacement data (d). In each figure, the slices at the seabed, $z=0.62 \mathrm{~km}$ and $y=0.42 \mathrm{~km}$ are shown
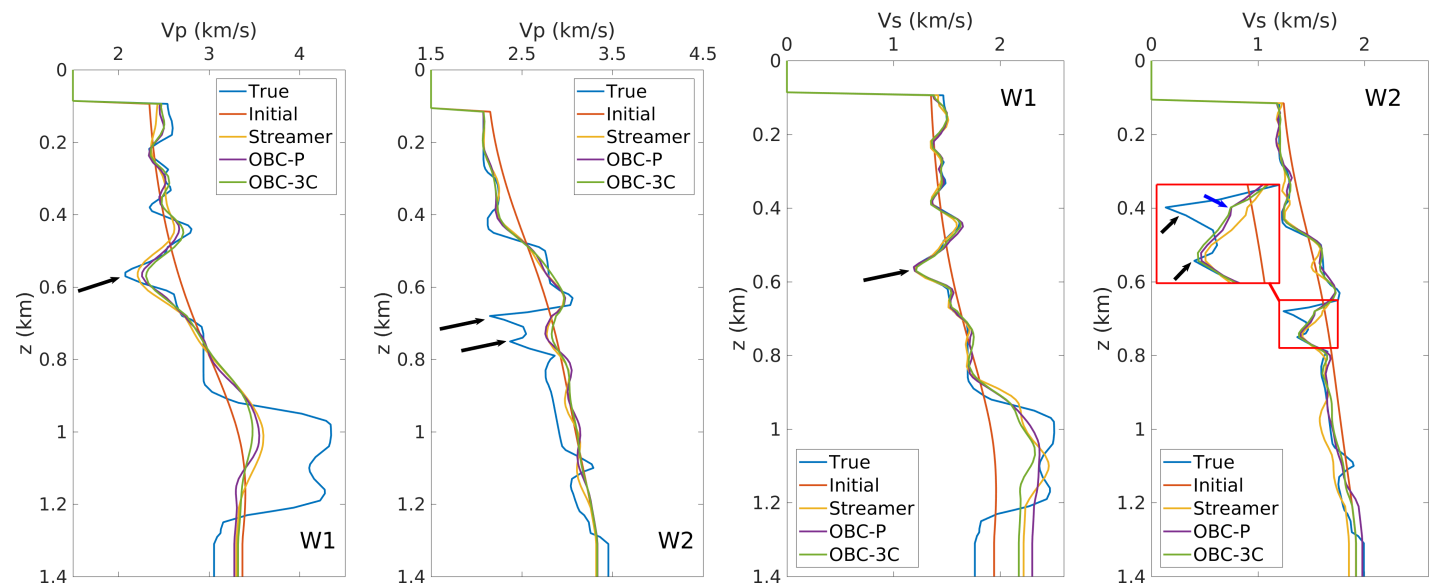

(a)

Figure 5: Vertical profiles for comparing the $V_{p}$ (a) and $V_{s}$ (b) inversion results at well locations $\mathrm{W} 1$ and $\mathrm{W} 2$. The low-velocity anomalies indicated by black arrows are gas traps. The inset in (b) shows zoom-in of the red square region which delineates two adjacent gas traps. 